\title{
Letter
}

Chun-Hui Miao*

\section{Why Don't Prices Rise during Periods of Peak Demand? Synchronize Demand to Relax Competition}

DOI 10.1515/bejeap-2015-0238

Published online June 3, 2016

\begin{abstract}
During periods of peak demand, frequent markdowns present an empirical puzzle. Based on the idea that stores face capacity constraints in times of high shopping volume, we show that stores keep their off-season prices high in order to lure all consumers to shop around the same time. This relaxes competition and allows stores to raise prices. Due to binding capacity constraints, stores randomize their prices. Thus, our model offers a unified explanation for both the countercyclicality and the high frequency of price changes during periods of peak demand.
\end{abstract}

Keywords: capacity constraint, peak demand, price competition JEL Classification: D00, L00, L40

A large body of empirical literature finds that prices are frequently marked down during periods of peak demand. This represents a puzzle to classic models of price determination in competitive markets, which predict that higher demands lead to either no change or an increase in prices. In this paper, we provide a novel explanation for the puzzle. We show that firms keep their off-season prices high in order to shift demand to the period of peak demand when their capacity constraints are binding, thereby relaxing competition and generating higher profits. Surprisingly, no firm can profitably deviate from the high off-season price. Furthermore, we predict that firms employ mixed pricing strategies during periods of peak demand. This prediction is consistent with the known pattern of sales on weekends and at holiday periods.

Warner and Barsky (1995) (henceforth WB-95) used survey data to investigate a wide variety of goods and found that "Individual prices change very often, and by nontrivial amounts" in the intensive shopping periods during the weekend and

*Corresponding author: Chun-Hui Miao, Department of Economics, University of South Carolina, Columbia, SC 29208, USA, E-mail: miao@moore.sc.edu 
before Christmas. MacDonald (2000) studied grocery prices and found many items whose price declined in periods when the total quantity purchased was high. Their findings were further confirmed by Chevalier, Kashyap and Rossi (2003) (henceforth CKR-03) using evidence from scanner data.

CKR-03 also developed an empirical strategy to test three theories of imperfect competition that can produce countercyclical prices. WB-95 conjectured that demand elasticities were cyclical due to economies of scale in search. Procyclical patterns of demand elasticities then produce countercyclical patterns of price markups. Rotemberg and Saloner (1986) suggested that countercyclical markups could result from changes in the ability of firms to sustain tacit collusion in situations where demand was changing from period to period. However, CKR-03 found that the evidence was most consistent with "loss-leader" models of advertising (Lal and Matutes 1994), in which retailers compete for customers via advertised prices and it is efficient for retailers to advertise and discount items in high relative demand.

In a subsequent study, Nevo and Hatzitaskos (2005) reexamined the CKR-03 data by using a fixed-weight price index. They found evidence more consistent with a change in price sensitivity and brand preferences, but less consistent with a theory of "loss leaders." Guler, Misra and Vilcassim (2014) introduced a model of consumer heterogeneity to show that, if a market is composed of a small segment with a high willingness to pay (WTP) and a big segment with a low WTP, there can be cases in which prices must fall in order to target the bigger segment during high demand. ${ }^{1}$ Bayot and Caminade (2014) examined the role of changes in aggregate elasticity during high demand periods and found that changes to aggregate elasticity were driven by the entry of a large share of new consumers that are more price elastic.

To the best of our knowledge, while the existing theories in the literature offered varying explanations why markups may be countercyclical, none of them explained the high frequency of price changes during periods of peak demand. The main novelty of this paper is to provide a unified theory that accounts for both observed features of the data.

\section{The Model}

In this market, a continuum of risk-neutral consumers have unit demands for a holiday good, valued at $v$. Following Chevalier and Kashyap (2014), we assume that some consumers are inactive shoppers that do not strategically time their

1 Their model also differs from ours in that they assume a monopolist retailer. 
purchases, while others do. More specifically, a measure of $\alpha$ consumers only shop in the holiday period, while $\beta$ consumers are bargain hunters that may make an advance purchase if they find a lower price preholiday.

Two stores $(i=A, B)$ sell the good over two periods, $t=1,2$, corresponding to preholiday and holiday. ${ }^{2}$ The good can be produced at a constant marginal cost of $c$, but a store can sell only a limited quantity of the good (or equivalently, serve a limited number of customers) in each period. ${ }^{3}$

Formally, each store's marginal cost function is

$$
C^{\prime}\left(q_{t}^{i}\right)=\left\{\begin{array}{ccc}
c & \text { if } q_{t}^{i} \leq k \\
+\infty & \text { if } q_{t}^{i}>k
\end{array} \text { for } i=A, B,\right.
$$

where $q_{t}^{i}$ is the number of units sold by store $i$ at time $t$ and $k$ is the maximum number of units available at each store per period. In order to streamline our exposition and focus on our main result, we further assume that $k \geq \beta$, which means that stores' capacity constraints are never binding during preholiday. Let $p_{t}^{i}$ denote the price and $\pi_{t}^{i}=\left(p_{t}^{i}-c\right) q_{t}^{i}$ the profit of store $i$ at time $t$, then stores compete in prices (a la Bertrand) to maximize their total profits: ${ }^{4}$

$$
\pi^{i}=\sum_{t=1}^{2} \pi_{t}^{i}=\sum_{t=1}^{2}\left(p_{t}^{i}-c\right) q_{t}^{i} \text { for } i=A, B .
$$

In each period, after stores simultaneously set prices, shoppers simultaneously make their purchase decisions. To concentrate on the particular type of shopping friction, i. e., some shoppers' inability to switch shopping channels, and have as stark a contrast as possible to the standard result of Bertrand competition, we abstract away from considerations of other types of shopping frictions such as search costs. Specifically, we assume that all shoppers are perfectly informed about the prices charged in the current period and that bargain hunters can correctly anticipate the holiday price (distribution). Thus, due to their risk-neutrality and unit demands, bargain hunters select the period to shop by comparing the preholiday price and the (expected) holiday price. If they are indifferent, then a fraction $x$ of bargain hunters choose to shop in the

2 To reduce the number of notations, we assume that there is no discounting between periods, but it is not difficult to see that adding a discount factor does not change our qualitative result. 3 Anecdotes are abundant. To quote from Debra Farmer, manager of a large supermarket, "During regular weeks, we restock the shelves during late night and early morning hours. But during these holidays, we have to do it every hour; we do not have enough manpower to do that" (Levy et al. 2010).

4 Albeit unrealistic, the assumption of price competition with homogeneous goods allows us to draw the sharpest contrast with the standard result of Bertrand competition. 
holiday period. By definition, $x \in[0,1]$, but its equilibrium value will be determined endogenously. During the shopping period, each shopper buys from the store that offers the lower price. If the two stores charge the same price in any given period, then each sells to half of the shoppers. If more than $k$ shoppers visit the same store, then they each have an equal probability of making a successful purchase. ${ }^{5}$ Shoppers that are turned away by the first store make purchases from the other store. The game equilibrium is characterized by stores' pricing strategies $\left\{p_{t}^{i}\right\}$ and $x$. Our solution concept is the subgame-perfect equilibrium.

\subsection{Holiday Pricing}

We start by solving the holiday subgame. Let $n \in R^{+}$denote the number of shoppers.

\section{Lemma 1:}

(i) For $n \in(0, k]$, there is a unique pure strategy equilibrium in which $p_{2}^{i}=c$, $\pi_{2}^{i}=0$, for $i=A, B$; for $n \in[2 k, \infty)$, there is a unique pure strategy equilibrium in which $p_{2}^{i}=v, \pi_{2}^{i}=(v-c) n / 2$, for $i=A, B$;

(ii) For $n \in(k, 2 k)$, there is a unique symmetric mixed-strategy equilibrium in which prices are distributed on the support of $[(v-c)(n-k) / k, v]$ with the cumulative distribution function (C.D.F). given by $F^{i}(p)=\left(\frac{(v-c)(n-k)}{p}-k\right) /$ $(n-2 k)$, for $i=A, B$. The expected price paid by a shopper is $E\left(p_{2}\right)=$ $c+2(v-c)(n-k) / n$. Each store earns a positive profit of $\pi_{2}^{i}=(v-c)(n-k)$.

Proof: In the appendix.

Lemma 1 shows that stores earn higher profits when their capacity constraints are binding. This is because shoppers turned away by one store become the other's captive consumers. Facing a trade-off between lowering the price to undercut the rival and raising the price to exploit the captive consumers, stores randomize their prices and earn positive profits. ${ }^{6}$ In other words, they gain from having all shoppers make purchases in the holiday period. It gives us the basic

5 In other words, we assume proportional rationing, but the efficient rationing rule generates the same rationing outcome in this model because consumers have the same reservation value. 6 The proof of case (ii) follows a similar one by Levitan and Shubik (1972), which solves a mixed-strategy equilibrium for capacity-constrained duopoly in the case of linear demands. 
intuition why stores may want to keep the preholiday price high and shift consumer demand to the holiday.

It is worth noting that, in case (ii) the average holiday price has a simple expression, even though the expected low price and high price take rather complicated forms in the mixed-strategy equilibrium. This is due to our assumption of unit demands: as long as every shopper makes a purchase, there is no aggregate welfare loss. Hence, the total surplus must be $n(v-c)$, which is the sum of store profits and consumer surplus (CS). Although stores randomize their prices, their profit function has a simple form, i. e., $(v-c)(n-k)$. Plugging it into the equation, we can easily calculate the total CS, which must equal $n(v-c)-2(v-c)(n-k)$. On average, the CS per shopper can thus be written as $\mathrm{CS} / n=(v-c)-2(v-c)(n-k) / n$. This is as if each shopper pays an expected price of $c+2(v-c)(n-k) / n$.

\subsection{Preholiday}

In the preholiday period, a marginal cost pricing equilibrium $\left(p_{1}^{A}=p_{1}^{B}=c\right)$ always exists, in which all bargain hunters make advance purchases. Its existence follows from the classic Bertrand competition argument: price undercutting leads to profit dissipation; at the same time, it is an equilibrium because bargain hunters cannot gain by delaying their purchases. The main concern of our analysis, however, will be the existence of other equilibria. In particular, we want to examine whether the pricing strategy $p_{1}>E\left(p_{2}\right)$ can be sustained in an equilibrium. In order to highlight our main result and illustrate the basic intuition, we start our analysis by considering a limited range of parameter values, $k \in\left[\frac{\alpha+\beta}{2}, \alpha\right]$, and then complete it by examining the entire range of parameter values.

\subsubsection{Main Result}

To pin down the equilibrium preholiday prices, we first determine $x$ as a function of $p_{1}^{\min }=\min \left\{p_{1}^{A}, p_{1}^{B}\right\}$, the preholiday market price. According to Lemma 1 , $E\left(p_{2}\right)=\max [c+2(v-c)(x \beta+\alpha-k) /(x \beta+\alpha), c] .{ }^{7}$ It is thus easy to see that

- If $p_{1}^{\min }>E\left(p_{2} \mid x=1\right)$, then $x=1$;

- If $p_{1}^{\min }<E\left(p_{2} \mid x=0\right)$, then $x=0$;

- If $p_{1}^{\min } \in\left[E\left(p_{2} \mid x=0\right), E\left(p_{2} \mid x=1\right)\right]$, then we can rule out the possibilities that $p_{1}^{\min }<E\left(p_{2}\right)$ (in which case $x=0$, contradiction) and $p_{1}^{\min }>E\left(p_{2}\right)$ (in which

7 It is easy to verify that $c+2(v-c)(x \beta+\alpha-k) /(x \beta+\alpha) \leq v$ when $k \geq \frac{\alpha+\beta}{2}$. 
case $x=1$, contradiction), so we must have $p_{1}^{\min }=E\left(p_{2}\right)>c$. This means that $x$ is the solution to $2(v-c)(x \beta+\alpha-k) /(x \beta+\alpha)=p_{1}^{\min }$. Note that $x$ is continuously increasing in $p_{1}^{\min }$ on the interval of $\left[E\left(p_{2} \mid x=0\right), E\left(p_{2} \mid x=1\right)\right]$.

Next we determine stores' profits as functions of their preholiday prices. Because of symmetry, we only need to compute $\pi^{A}$. We divide the analysis into two cases:

(i) $c<p_{1}^{B}<E\left(p_{2} \mid x=1\right)$ - the rival sets a low preholiday price to attract bargain hunters:

- If $p_{1}^{A}>p_{1}^{B}$, then $p_{1}^{\min }=p_{1}^{B}$, store $A$ has customers only in the holiday period, so $\pi^{A}=\max [(v-c)(x \beta+\alpha-k), 0]$, which does not vary with $p_{1}^{A}$;

- $\quad$ if $p_{1}^{A}=p_{1}^{B}$, then $p_{1}^{\min }=p_{1}^{A}$, so $\pi^{A}=(1-\chi) \beta\left(p_{1}^{A}-c\right) / 2+(v-c)(x \beta+\alpha-k)$;

- if $p_{1}^{A}<p_{1}^{B}$, then $p_{1}^{\min }=p_{1}^{A}$ and $x<1$, so $\pi^{A}=(1-x) \beta\left(p_{1}^{A}-c\right)+(v-c)$ $(x \beta+\alpha-k)$ and $\frac{\partial}{\partial p_{1}} \pi^{A}=\beta(1-x)+\beta \frac{\partial x}{\partial p_{1}}\left(v-p_{1}^{A}\right)>0$.

(ii) $p_{1}^{B} \geq E\left(p_{2} \mid x=1\right)$ - the rival sets a high preholiday price:

- if $p_{1}^{A}<E\left(p_{2} \mid x=1\right)$, then $p_{1}^{A}<p_{1}^{B}, p_{1}^{\min }=p_{1}^{A}$, and $x<1$, so $\pi^{A}=(1-x)$ $\beta\left(p_{1}^{A}-c\right)+(v-c)(x \beta+\alpha-k)$ and $\frac{\partial}{\partial p_{1}} \pi^{A}=\beta(1-x)+\beta \frac{\partial x}{\partial p_{1}}\left(v-p_{1}^{A}\right)>0 ;$

- If $p_{1}^{A} \geq E\left(p_{2} \mid x=1\right)$, then $p_{1}^{\min } \geq E\left(p_{2} \mid x=1\right)$ and $x=1$, store $A$ has customers only at time 2 , so $\pi^{A}=(v-c)(\alpha+\beta-k)$. This means that $\pi^{A}$ increases with $p_{1}^{A}$ up to $E\left(p_{2} \mid x=1\right)$, but does not increase any further.

Summarizing, store $A$ 's best response is to undercut the rival, i.e., $p_{1}^{A}\left(p_{1}^{B}\right)=p_{1}^{B}-\varepsilon$, if $c<p_{1}^{B}<E\left(p_{2} \mid x=1\right)$; or to keep the price high, i. e., $p_{1}^{A}\left(p_{1}^{B}\right) \in$ $\left.E\left(p_{2} \mid x=1\right), \infty\right)$, if $p_{1}^{B} \geq E\left(p_{2} \mid x=1\right)$. It is also easy to see that $\left.p_{1}^{A}\left(p_{1}^{B}=c\right) \in c, \infty\right)$. Therefore, we can conclude that

Proposition 1: If $k \in\left[\frac{\alpha+\beta}{2}, \alpha\right]$, then there are two subgame-perfect equilibria:

(i) A splitting equilibrium $(x=0): p_{1}^{i}=c, p_{2}^{i} \in[c+(v-c)(\alpha-k) / k, v]$ with the C.D.F. given by $F^{i}(p)=\left(\frac{(v-c)(\alpha-k)}{p-c}-k\right) /(\alpha-2 k), i=A, B$, and $E\left(p_{2}\right)=c+2$ $(v-c)(\alpha-k) / k$.

(ii) A crowding equilibrium $(x=1): p_{1}^{i} \geq c+2(v-c)(\alpha+\beta-k) / k, p_{2}^{i} \in[c+(v-c)$ $(\alpha+\beta-k) / k, v]$ with the C.D.F. given by $F^{i}(p)=\left(\frac{(v-c)(\alpha+\beta-k)}{p-c}-k\right) /$ $(\alpha+\beta-2 k), i=A, B$, and $E\left(p_{2}\right)=c+2(v-c)(\alpha+\beta-k) / k$.

(iii) For the stores, the crowding equilibrium Pareto dominates the splitting equilibrium.

In a splitting equilibrium, bargain hunters stores' capacity constraints are binding only in the holiday period, the period of peak demand. This naturally 
leads to procyclical pricing, i. e., price rising at the time of peak demand. ${ }^{8}$ More interesting, however, is the existence of a crowding equilibrium, in which stores keep the preholiday price so high that all shoppers choose to make purchases in the holiday period. This is in stark contrast to the standard marginal cost pricing result under Bertrand competition, based on the usual price undercutting argument. The intuition why high preholiday prices can be sustained as an equilibrium outcome in our model is as follows. In a candidate crowding equilibrium, we must have $p_{1} \geq E\left(p_{2}\right)$. For (preholiday) price cutting to possibly be a profitable deviation, a store must set $p_{1} \in\left(c, E\left(p_{2}\right)\right]$. Now suppose that some bargain hunters do switch to preholiday purchases because of the lower $p_{1}$, their number must be so small that the holiday capacity constraint is still binding, for otherwise $E\left(p_{2}\right)$ will become $c$, contradicting the bargain hunters' choice of switching to preholiday shopping. This means that, for every marginal shopper that it attracts, a store gains $p_{1}$ on the preholiday but loses $v$ in the holiday period. Since $p_{1}<E\left(p_{2}\right)<v$, neither store has a profitable deviation in lowering $p_{1} \cdot{ }^{9}$

The crowding equilibrium contains two important features of the empirical evidence that have been previously documented: first, even though the holiday is the period of peak demand, its price on average does not rise above the preholiday price; second, correctly anticipated by the bargain hunters, stores randomize their prices in the holiday period, generating a pattern of sales consistent with what WB95 calls "a simple, predictable, and publicly known patterns of sales on weekends, at holiday periods, and (especially) on holiday weekends, that are concentrated at mall and outlet stores and take place at all or most stores simultaneously." This distinguishes our model from information-based theories of sales, which are costly to detect and thus unpredictable in time or space.

\subsubsection{Full Results}

To complete the analysis, we now examine the remaining ranges of parameter values. If each store's holiday capacity $k$ is greater than $\alpha+\beta$, then a single store can serve all shoppers. It is immediate from case $(i)$ of Lemma 1 that the game outcome must be the Bertrand outcome, in which both stores price the good at marginal cost and earn zero profits. What if $k \in \alpha, \alpha+\beta]$ ? According to the above

8 Thus, our model can generate predictions of both procyclical pricing and countercyclical pricing. As summarized in Table 1, which one of the two is observed largely depends on the severity of capacity constraints that stores face.

9 The same argument can be used to extend our result easily to the case of oligopoly, where $M>2$ stores compete. 
Table 1: Equilibrium Values Under Different Capacity Levels.

\begin{tabular}{llll}
\hline Conditions & $\boldsymbol{p}_{\mathbf{1}}$ & $\boldsymbol{E}\left(\boldsymbol{p}_{2}\right)$ & $\mathbf{x}$ \\
\hline$k \in\left(0, \frac{\alpha+\beta}{2}\right)$ & $c$ & $\min \left\{c+\frac{2(v-c)(\alpha-k)}{\alpha}, v\right\}$ & 0 \\
$k \in\left[\frac{\alpha+\beta}{2}, \alpha\right)$ & $c+\left[\frac{2(v-c)(\alpha+\beta-k)}{\alpha+\beta}, \infty\right)$ & $c+\frac{2(v-c)(\alpha+\beta-k)}{\alpha+\beta}$ & 1 \\
$k \in[\alpha, \alpha+\beta)$ & $c+\left[\frac{2(v-c)(\alpha+\beta-k)}{\alpha+\beta}, \infty\right)$ & $c+\frac{2(v-c)(\alpha-k)}{\alpha}$ & 0 \\
$k \in[\alpha+\beta, \infty)$ & $c$ & $c$ & {$\left[0, \frac{k-\alpha}{\beta}\right]$} \\
\hline
\end{tabular}

analysis, it is now possible to have a partial crowding equilibrium, in which $x \leq(k-\alpha) / \beta$; at the same time, a crowding equilibrium still exists.

Last, we find that the unique equilibrium is a splitting one if $k<\frac{\alpha+\beta}{2}$. To see why, note that $E\left(p_{2}\right)=\min [c+2(v-c)(x \beta+\alpha-k) /(x \beta+\alpha), v]$, hence $E\left(p_{2} \mid x=1\right)=v$. This means that, in order to sustain the crowding equilibrium, we must have $p_{2}^{i}=v$ and $p_{1}^{i} \geq v$. Thus, $\pi^{A}=(v-c) k$. But if store $A$ lowers its price such that $p_{1}^{A}=v-\varepsilon$, then $\pi^{A}=\beta(v-\varepsilon-c)+(v-c)(\alpha-k)>k(v-c)$ : there is a profitable deviation. A partial crowding equilibrium does not exist either, because an infinitesimal decrease in $p_{1}^{A}$ leads to a gain of $(1-x) / 2$ bargain hunters.

\section{Extensions}

In this section, I consider extensions of the basic model to check robustness of the results.

\subsection{Randomly Distributed Reservation Prices}

Consider a simple extension of the basic model: suppose that consumers' valuations are independent of type but are randomly distributed on the support of $[\underline{v}, \bar{v}]$ according to $F(v)$. Suppose further that the demand function thus formed satisfies the properties given in Allen and Hellwig (1993) so that a holiday subgame equilibrium exists and has the following property:

Assumption 1: If $F(v) \leq G(v)$ for all $v$, then $\Pi^{F} \geq \Pi^{G}$, where $\Pi^{F}$ (respectively, $\Pi^{G}$ ) is the industry profit if shoppers' valuations are drawn from the distribution $F(v)$ (respectively, G(v)). 
We argue below that our main result that a crowding equilibrium exists continues to hold as long as the market is covered in the equilibrium. In order to break the crowding equilibrium, a store must cut its preholiday price to $p_{1}^{\prime} \in\left[c, E\left(p_{2}^{\prime}\right)\right]$ such that $\chi^{\prime}<1$, but the stores' holiday capacity constraints must still be binding, for otherwise $E\left(p_{2}^{\prime}\right)=c \leq p_{1}^{\prime}{ }^{10}$ This means that the number of remaining consumers, $\beta x^{\prime}+\alpha$, is greater than $k$. Note that, however, the price cut only attracts consumers with a minimum valuation of $p_{1}^{\prime}$, so the distribution of remaining consumers is first-order stochastically dominated by the original distribution $F(v){ }^{11}$ The deviating store's period 1 gain is at most $\beta\left(1-x^{\prime}\right)\left[E\left(p_{2}^{\prime}\right)-c\right]$, while its period 2 loss is $\pi_{2}-\pi_{2}^{\prime}$. Because of the symmetry between stores, we must have $\pi_{2}^{\prime}=\Pi_{2}^{\prime} / 2$, where $\Pi_{2}^{\prime}=\left(\beta x^{\prime}+\alpha\right)\left[E\left(p_{2}^{\prime}\right)-c\right]$ is the industry profit at $t=2$ if the market is covered. Let $\hat{\Pi}_{2}$ denote the industry profit from $\beta x^{\prime}+\alpha$ shoppers, whose valuations are drawn from the original distribution $F(v)$. By Assumption 1, $\hat{\Pi}_{2} \geq \Pi_{2}^{\prime}$. At the same time, $\frac{\hat{\Pi}_{2} / 2}{\pi_{2}}=\frac{\beta x^{\prime}+\alpha-k}{\beta+\alpha-k}$. Therefore, the net gain for the deviating store is

$$
\begin{aligned}
\beta\left(1-x^{\prime}\right)\left[E\left(p_{2}^{\prime}\right)-c\right]-\left(\pi_{2}-\pi_{2}^{\prime}\right)= & \frac{\beta\left(1-x^{\prime}\right) \Pi_{2}^{\prime}}{\left(\beta x^{\prime}+\alpha\right)}-\frac{\hat{\Pi}_{2}}{2} \frac{\beta+\alpha-k}{\beta x^{\prime}+\alpha-k} \\
& +\frac{\Pi_{2}^{\prime}}{2} \leq \frac{\beta\left(1-x^{\prime}\right)}{2} \frac{\beta x^{\prime}+\alpha-2 k}{\left(\beta x^{\prime}+\alpha\right)\left(\beta x^{\prime}+\alpha-k\right)} \hat{\Pi}_{2} \leq 0
\end{aligned}
$$

as long as $k \geq \frac{\alpha+\beta}{2}$.

\subsection{Infinitely lived Market}

So far we have assumed that some consumers only shop at time 2, which is the last stage of the game. This means that peak demand may be realized in the late stage, but not the early. Moreover, a market that lasts only two periods is clearly unrealistic. In the following, we show that it is straightforward to extend our model to have infinitely many periods, during which consumers make repeated purchases.

Consider a repeated version of the game studied in the basic model. The game lasts for an infinite number of periods. Each shopper has a unit demand for every two periods. While bargain hunters can make purchases in any period, holiday shoppers can only make purchases in an even-numbered period. We rule out punishment strategies by assuming prices cannot be conditioned upon

10 All symbols with the apostrophe denote the new values after the price-cutting deviation. 11 This makes it exceedingly difficult to pin down the equilibrium price distribution (even in simple cases such as uniformly distributed $v$ ) or compute $E\left(p_{2}^{\prime}\right)$, unless the market is covered. 
history to ensure that our result is not driven by the existence of repeated interactions among firms such as in Rotemberg and Saloner (1986). It is immediately clear that, if the game starts at $t=1$, then the above game is just a simple repetition of the two-period model. All results remain the same as in the basic model. What if the game starts at $t=0$ ? It makes little difference: since the market is covered in a crowding equilibrium, everyone will have made purchases by the end of $t=2$ and therefore, starting from $t=3$, the game is again a simple repetition of the two-period model.

\subsection{Endogenous Choice of Capacities}

In the above model, stores compete under exogenous capacity constraints. Here we discuss what happens if stores can build capacities before they compete. ${ }^{12}$ Because of the equilibrium multiplicity in the stage game, the capacity choices hinge upon the refinement criteria being used. The following discussion is based on the Pareto dominance criteria of equilibrium selection. Let $k^{A}$ (respectively, $k^{B}$ ) denote the holiday capacity of store $A$ (respectively, $B$ ). We assume that capacity is costly (e.g., for each unit of capacity, it costs $\varepsilon$ to install), but not too costly $(\varepsilon<v-c)$. It naturally follows that a store will choose the smallest possible capacity that can sustain a crowding equilibrium (If we limit our attention to symmetric equilibria, then each store will choose a capacity of $\frac{\alpha+\beta}{2}$ ). To see why, note that as long as a crowding equilibrium is obtained, a store (say, store $i$ ) does not gain by changing its capacity, since its profit in any crowding equilibrium is always $\left(\alpha+\beta-k^{j}\right)(v-c)$, which decreases with the rival's capacity but does not vary with its own capacity $k^{i}$. At the same time, the capacities chosen must be sufficiently large, otherwise stores cannot obtain the more profitable crowding equilibrium, as shown in Table 1. In sum, when capacities are endogenously chosen, they will indeed fall into the range for which stores find it profitable to synchronize demand. ${ }^{13}$

\section{Conclusion}

During periods of peak demand, frequent markdowns present an empirical puzzle. While existing theories in the literature have offered varying explanations why markups may be countercyclical, none of them explains the high

12 I am grateful to an anonymous referee for suggesting this analysis.

13 A formal proof is in the online appendix. 
frequency of price changes. This paper provides a unified theory that accounts for both observed features of the data.

\section{Appendix: Proof}

Proof of Lemma 1: (i) is obvious; (ii) For notational simplicity, we omit the time subscript $t$ in the proof. Let $F^{j}(p)$ denote the C.D.F. of the price of firm $j$. Thus, with a probability of $1-F^{j}\left(p^{i}\right)$, firm $i$ has a lower price and sells its capacity of $k$; with a probability of $F^{j}\left(p^{i}\right)$, firm $i$ has a higher price and serves only the residual demand of $n-k$. Hence, firm ís expected quantity sold is $E\left(q^{i}\right)=\left[1-F^{j}\left(p^{i}\right)\right] k+F^{j}\left(p^{i}\right)(n-k)$. Its expected profit is $\pi^{i}=\left(p^{i}-c\right) E\left(q^{i}\right)=\left(p^{i}-c\right)\left[k+F^{j}\left(p^{i}\right)(n-2 k)\right]$. It is easy to see that upper bound of the equilibrium distribution of price is $\bar{p}=v$, acting as a monopolist on the residual demand. Thus, $\pi^{i}=\pi^{i}(\bar{p})=(\bar{p}-c)(n-k)=(v-c)(n-k)$. Let $\underline{p}$ denote the lower bound of the price distribution.

First, we show that $F^{A}(p)$ is atomless in the interior of $[p, \bar{p}]$. Suppose that firm $A$ names the price at $p \in(p, \bar{p})$ with some positive probability $q$, then firm $B$ cannot be indifferent between $p$ and $p+\varepsilon$ : when firm $B$ changes its price from $p+\varepsilon$ to $p$, it increases its sales by $q / 2$ but lowers its average price in the order of $\varepsilon$. Therefore, it must strictly prefer $p$ over $p+\varepsilon$. This means that $p+\varepsilon$ cannot be on the support of $[\underline{p}, \bar{p}]$, since a player must be indifferent among the prices which it mixes over given its opponent's price distribution. In other words, $p$ cannot belong to the interior of $[p, \bar{p}]$. By similar reasoning, one can show that $F^{B}(p)$ is also atomless in the interior of $[p, \bar{p}]$ and that there is no equilibrium in pure strategies.

Next, we solve for the equilibrium price distribution. When a store sets $\underline{p}$, it sells to shoppers up to its capacity $k$ and its profit is $k(\underline{p}-c)$. Thus, we must have $\underline{p}=c+(v-c)(n-k) / k$. From the profit function, we can also get

$$
F^{j}\left(p^{i}\right)=\left(\frac{(v-c)(n-k)}{p^{i}-c}-k\right) /(n-2 k) .
$$

Because of symmetry,

$$
F^{i}(p) \equiv F^{j}(p)=\left(\frac{(v-c)(n-k)}{p-c}-k\right) /(n-2 k) .
$$

Hence, $f(p)=-\frac{v-c}{(p-c)^{2}} \frac{n-k}{n-2 k}$. 
Last, we calculate the expected price paid by a shopper. Each shopper sees the realization of two prices, one from each store. Due to stores' capacity constraints, only $k$ shoppers can make purchases at the lower price, which has a density function of

$$
f^{\min }(p)=2(1-F(p)) f(p)=\frac{2(v-c)(n-k)^{2}(v-p)}{(p-c)^{3}(n-2 k)^{2}} .
$$

The remaining $1-k$ shoppers pay a higher price, which has a density function of

$$
f^{\max }(p)=2 F(p) f(p)=2 \frac{\frac{(v-c)(n-k)}{p-c}-k}{n-2 k} \frac{-(v-c)}{(p-c)^{2}} \frac{n-k}{n-2 k} .
$$

Therefore, each shopper pays

$$
E\left(p^{\min }\right)=\int_{\underline{p}}^{\bar{p}} p f^{\min }(p) d p=c+2(v-c)(n-k) \frac{(n-k) \ln \frac{n-k}{k}-(n-2 k)}{(n-2 k)^{2}}
$$

with a probability of $\frac{k}{n}$ and

$$
E\left(p^{\max }\right)=c+2(v-c)(n-k) \frac{n-2 k-k \ln \left(\frac{n-k}{k}\right)}{(2 k-n)^{2}}
$$

with a probability of $\frac{n-k}{n}$. Thus, on average, a shopper pays a price of $E(p)=c+\left[k E\left(p^{\min }\right)+(n-k) E\left(p^{\max }\right)\right] / n=c+2(v-c)(n-k) / n$.

Acknowledgments: The author is grateful to Adam Wong, Junjie Zhou, seminar participants at the 2015 International Industrial Organization Conference, the 2015 Southern Economic Meeting, Hunan University, Shanghai University of Finance and Economics, the University of Hong Kong, USC - Columbia, and an anonymous referee for helpful comments and suggestions. All remaining errors are mine.

\section{References}

Allen, B. and M. Hellwig. 1993. "Bertrand-Edgeworth Duopoly with Proportional Residual Demand." International Economic Review 34 (1):39-60.

Bayot, D. and J. Caminade. 2014. "Popping the Cork: Why the Price of Champagne Falls During the Holidays." Working paper, University of Chicago.

Chevalier, J. A. and A. K. Kashyap 2014. "Best prices." NBER working paper no. 20768. 
Chevalier, J. A., A. K. Kashyap and P. Rossi. 2003. "Why Don’t Prices Rise During Peak Demand Periods? Evidence from Scanner Data." American Economics Review 93 (1):15-37.

Guler, A. U., K. Misra and N. Vilcassim. 2014. "Countercyclical Pricing: A Consumer Heterogeneity Explanation.” Economics Letters 122 (2):343-7.

Lal, R. and C. Matutes. 1994. "Retail Pricing and Advertising Strategies." Journal of Business 67 (3):345-70.

Levitan, R. and M. Shubik. 1972. "Price Duopoly and Capacity Constraints." International Economic Review 13 (1):111-22.

Levy, D., H. Allan Chen, G. Müller, S. Dutta and M. Bergen. 2010. "Holiday Price Rigidity and Cost of Price Adjustment.” Economica 77 (305):172-98.

MacDonald, J. M. 2000. "Demand, Information, and Competition: Why Do Food Prices Fall at Seasonal Demand Peaks?" The Journal of Industrial Economics 48 (1):27-45.

Nevo, A. and K. Hatzitaskos. 2005. "Why Does the Average Price of Tuna Fall During Lent?" NBER working paper.

Rotemberg, J. J. and G. Saloner. 1986. "A Supergame-Theoretic Model of Price Wars during Booms.” American Economic Review 76 (3):390-407.

Warner, E. J. and R. B. Warner. 1995. "The Timing and Magnitude of Retail Store Markdowns: Evidence from Weekends and Holidays.” Quarterly Journal of Economics 110 (2):321-52. 